\title{
Mechanical Performance of Parallel Bamboo Strand Lumber Columns under Axial Compression: Experimental and Numerical Investigation
}

\author{
Cheng Tan ${ }^{\mathrm{a}, \mathrm{b}}$, Haitao $\mathrm{Li}^{\mathrm{a}^{*} \text {, Dongdong Weic, Rodolfo Lorenzo }}{ }^{\mathrm{d}}$, Conggan Yuan \\ ${ }^{a}$ Collage of Civil Engineering, Nanjing Forestry University, Nanjing, China. \\ ${ }^{\mathrm{b}}$ Department of Civil and Environmental Engineering, Syracuse University, Syracuse, USA. \\ ${ }^{c}$ Jiangxi Feiyu Bamboo Stock Co. LTD, Fengxin 330700, China. \\ ${ }^{\mathrm{d}}$ University College London, London WC1E 6BT, UK \\ *Corresponding Author: Haitao Li. Email: 1haitao1982@126.com.
}

\begin{abstract}
This paper presents an investigation on the mechanical performance of parallel bamboo strand lumber (PBSL) columns under axial compression. Experimental test and numerical analysis were performed for 40 PBSL columns with various slenderness ratios. Failure modes, ultimate capacity and load-strain response are reported and evaluated. Strength failure is the typical failure mode of columns with small slenderness ratios, however, buckling failure is commonly observed for longer columns. Elastic eigenvalue analysis is found effective to predict critical buckling load of long columns, as buckling occurs within elastic range. However inelastic behavior has significant effect on critical load when the buckling stress exceeds proportional limit of the material. As a result, inelastic approaches provide more accurate prediction of critical load for columns with a slenderness ratio lower than the elastic threshold $\left(\lambda_{y}\right)$. The presented experimental results and numerical analysis validated the feasibility of the elastic/inelastic buckling analysis approaches on determination of ultimate capacity of axial loaded PBSL columns.
\end{abstract}

Keywords: Parallel bamboo strand lumber, axial compression, buckling, inelastic analysis.

\section{Introduction}

As an environmental friendly material, bamboo has been widely used as construction materials due to its excellent mechanical behavior [1], renewability and fast growing characteristic [2,3]. Compared to conventional construction materials, such as concrete, steel, and aluminium alloy, the strength-to-weight of bamboo is relatively high [4], which allows bamboo to be an efficient alternative of construction material.

The application of unprocessed bamboo is limited by its natural characteristics such as limited dimension, irregular shape and poor rigidity $[5,6]$. These drawbacks can be alleviated by reassembling the bamboo culms into desired forms with hot-pressure and adhesives [1,7], which referred as engineered bamboo. The most widely used two types of engineered bamboo are parallel bamboo stand lumber (PBSL) and laminated bamboo. Extensive studies have been conducted for engineered bamboo materials in terms of mechanical properties and engineering applications [8-26].

Mechanical properties of PBSL is significantly affected by the manufacturing process, raw material selection, moisture content and resin properties [1,8-10]. In general, mechanical properties of PBSL are comparable to or surpass that of traditional wood products [6]. In addition to material property investigation [11], extensive studies have been conducted on PBSL structural members in order to develop fundamental design, analysis and construction guidelines. Cui et al. [12-13] experimentally investigated the flexural behavior of PBSL beams and Huang et al. [14] developed a numerial analytical model to predict the bending performance of PBSL beams. Li et al. $[5,15]$ investigated the mechanical performance of PBSL columns under eccentric loading, and proposed an eccentricity influencing coefficient to account for the eccentric effect. Huang et al. [16] proposed an iterative anlytical model to predict ultimate 
capacity of eccentrically loaded intermediate slenderness PBSL columns. Wang et al. [17] investigated the mechanical behavior of PBSL column under biaxial eccentric compression and developed an analytical model to predict the load-carrying capacity. Chen and Zhao [18-19] investigated the effect of holes with different shape, size and location on PBSL beams. Zhou et al.[20] performed an experimental study on the embeding strength of PBSL materials, test results shows the embedding strength is dominated by the bolt diameter. Cross-section size effect on compressive strength of PBSL columns was studied by Zhao [21] and a section-effect reduction factor was proposed.

There are a few studies on compressive behavior of PBSL columns, however, few study was conducted on buckling behavior of PBSL column with various slenderness ratios. In addition, current proposed critical load $\left(P_{c r}\right)$ analysis approaches of PBSL columns are based on linear elastic theory, non-linear effect on buckling behavior is rarely addressed or requires relative large amount of computational efforts.

This paper presents an experimental investigation of compressive behavior of PBSL columns with slenderness ratios ranging from 13.8 to 62.3 along with linear elastic and inelastic buckling analysis. Verification of the elastic/inelastic approach was made against experimental results.

\section{Experimental Tests}

The experimental test consists of eight groups of specimens with different length ( $400 \mathrm{~mm}, 600$ $\mathrm{mm}, 800 \mathrm{~mm}, 1000 \mathrm{~mm}, 1200 \mathrm{~mm}, 1400 \mathrm{~mm}, 1600 \mathrm{~mm}$ and1800 mm), as shown in Fig. 1. Each group consists of five identical specimens.

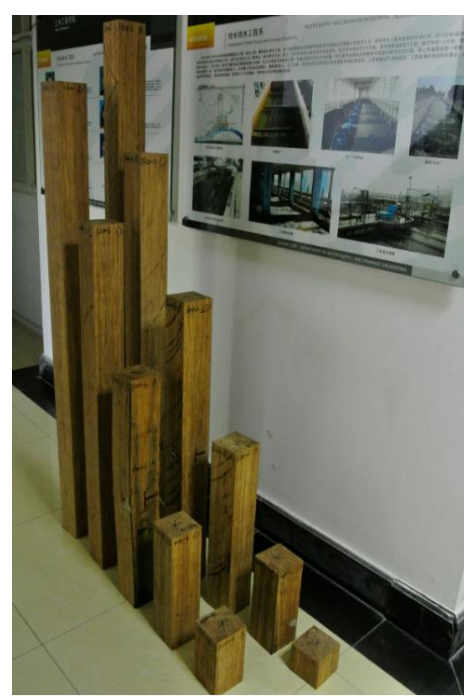

Fig. 1. PBSL columns with various length

\subsection{Materials}

The raw bamboo material was Moso bamboo from Jiangxi Province, China. Bamboo strips were split into filament bundles and then charred at a temperature of $165^{\circ} \mathrm{C}$ and a pressure of $0.3 \mathrm{MPa}$. The dried and charred bamboo filament bundles were formed into a rectangular shape with Phenolic adhesives under $90 \mathrm{MPa}$ transverse pressure and cured at a temperature of $140{ }^{\circ} \mathrm{C}$. The density of the PBSL was reported as $1018 \mathrm{~kg} / \mathrm{m}^{3}$ and the water content was $8 \pm 1 \%$ on the day of testing. Specimens were cut and polished at laboratory of Nanjing Forestry University. According to the compressive test, the peak strength $\left(f_{u}\right)$ of is $63.92 \mathrm{MPa}$ and the yield strength $\left(f_{y}\right)$ is $37.64 \mathrm{MPa}$. Compressive elastic modulus and Poisson's ratio are $11684.36 \mathrm{MPa}$ and 0.39 , respectively. Fig. 2. shows stress-strain relationship of the PBSL used in this study. 


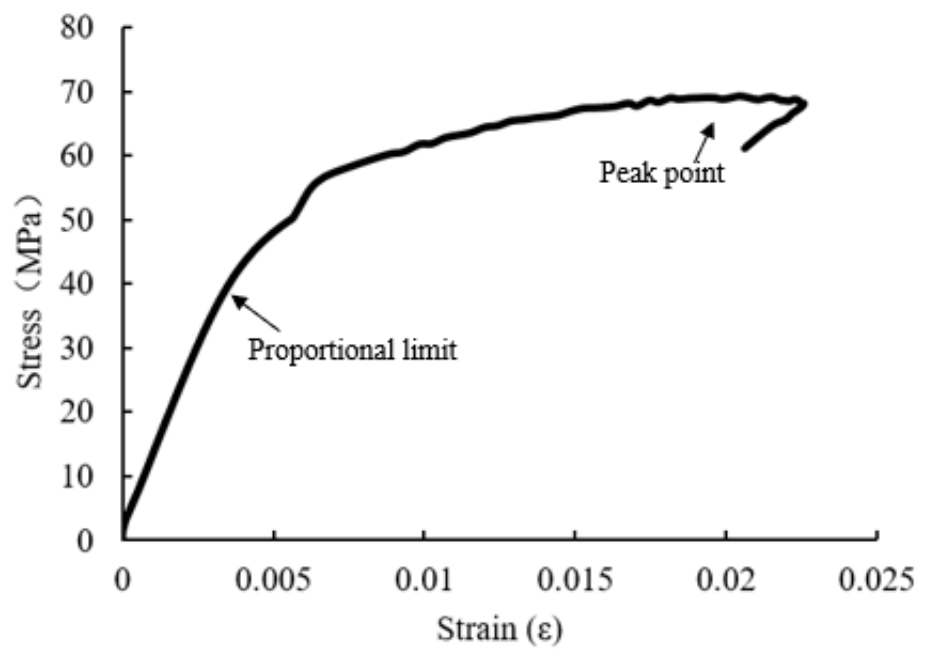

Fig. 2. Stress-strain relationship

\subsection{Test setup and instrumentation}

Pin support was selected for both ends, as shown in Fig. 3. The applied load was recorded by the built-in load cell of the $1000 \mathrm{kN}$ electro-hydraulic testing machine. Longitudinal displacement was measured with laser displacement sensors (LDS) on opposite sides of the columns, the average of the two longitudinal LDS data was adapted for analysis. In addition, lateral displacement of the columns on quarter points were measured with LDS, dash lines in Fig. 3 show the direction of lasers. Longitudinal and transverse strain at mid-height were measured with strain gauges on all the four surfaces. All data were collected by the TDS-530 data acquisition system. Fig. 4 shows the test setup of the column specimens.

Five cycles of pre-loading were performed for each specimen and specimen placement was adjusted until strain gauge on each surface showed similar values, which indicated the specimen was under pure axial loading. Load control was adapted before the columns reaching its linear proportional limit and it was switched to displacement control beyond the linear proportional limit at a rate of $3.6 \mathrm{~mm} / \mathrm{min}$.

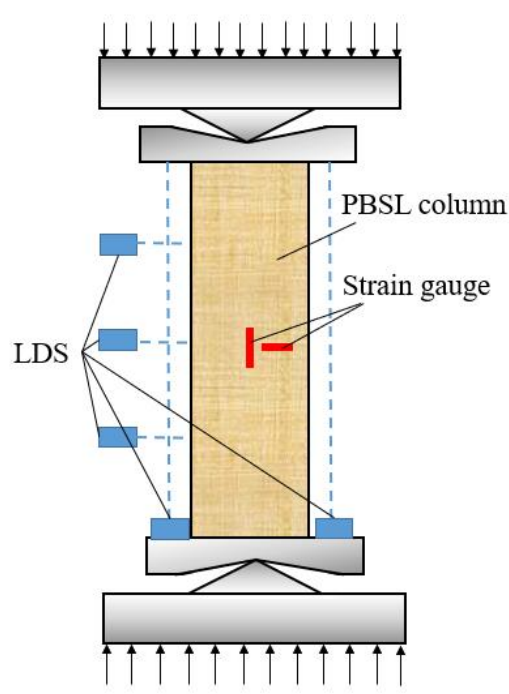

Fig. 3. Instrumentation

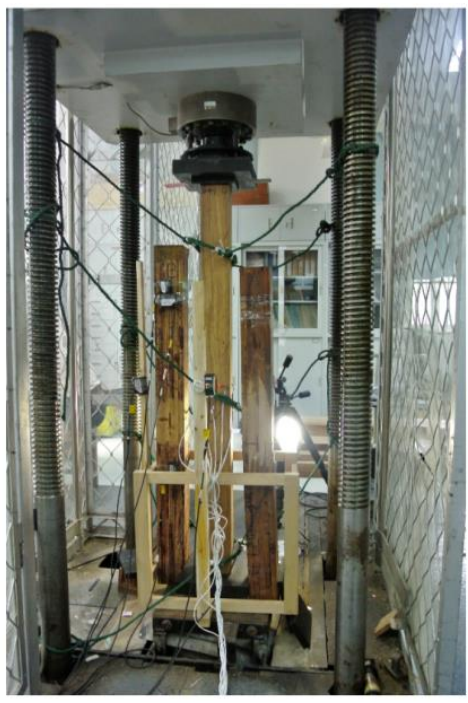

Fig. 4. Test setup

\section{Test Results and Discussion.}

\subsection{Failure mode and mechanism}


105

106

107

108

109

110

Strength failure, such as squashing or crushing of bamboo fibers, was the ultiamte failure mode of columns with a slenderness ratio $(\lambda)$ smaller than 17 , which referred as short columns [27]. Tested columns remained linear elastic until $400 \mathrm{kN}$, yielding took place before crushing/squashing of the column (Fig. 9). At the failure stage, diagonal dislocation occurred near the end of columns, with fibers outwards splitting at corners, as shown in Fig. 5. Similar failure mode was observed for $600 \mathrm{~mm}$ height columns $(\lambda=20.72)$.

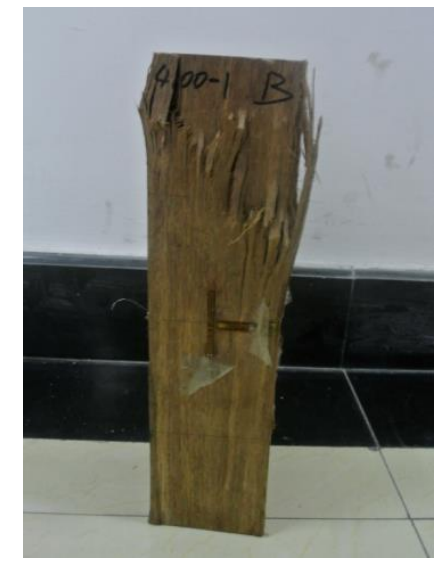

(a) Diagonal dislocation

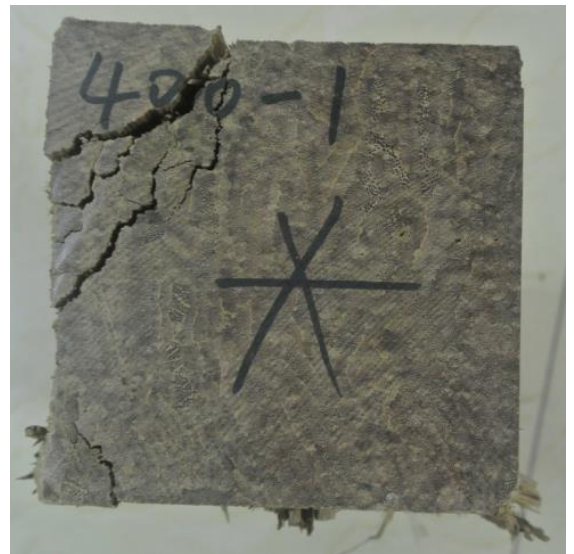

(b) Top surface

Fig. 5. Strength failure

\subsubsection{Buckling failure}

For columns with a height lager than $600 \mathrm{~mm}$, buckling dominated the ultimate failure, as shown in Fig. 6a. Large lateral deflection was observed at failure along with snapping of bamboo fibers at mid-height. For columns with a length ranging from 600-1400, apparent yielding before failure was observed (Fig. 9). However, for group $1600 \mathrm{~mm}$ and $1800 \mathrm{~mm}$, yielding was not observed before buckling (Fig. 9). A few specimen failed in buckling along with severe splitting of the column from one end, as shown in Fig. 6b. This is attributed to large lateral deflection formed at mid-height, whilelateral displacement was restrained at supports, internal stress was exerted to maintain compatibility.
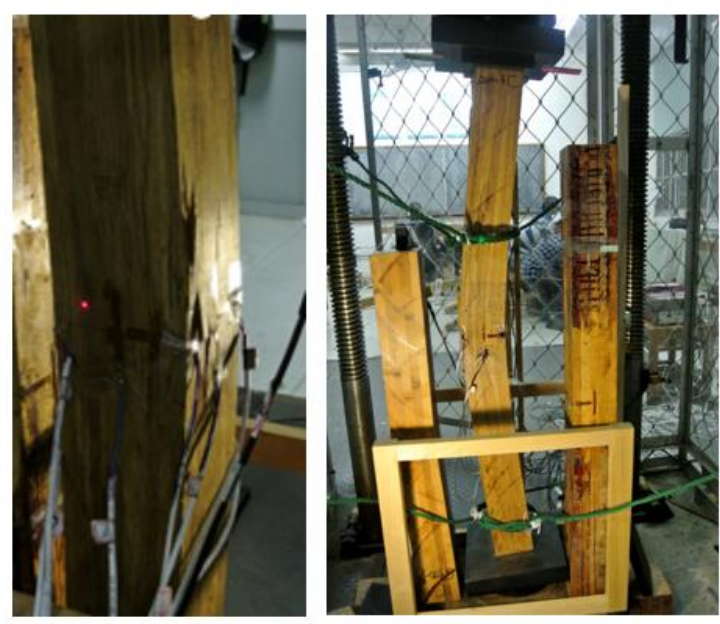

(a) Large lateral deflection

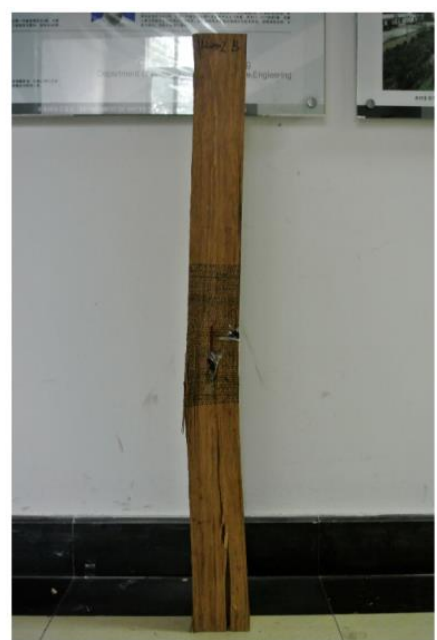

(b) Column splitting 
Fig. 7 shows load versus lateral deflection at mid-height for specimens with various length. For short columns, no significant deflection was observed until reaching its ultimate capacity. However, for long columns, apparent deflection was observed before bucking occurred. Load capacity dropped gradually beyond the peak load until buckling.

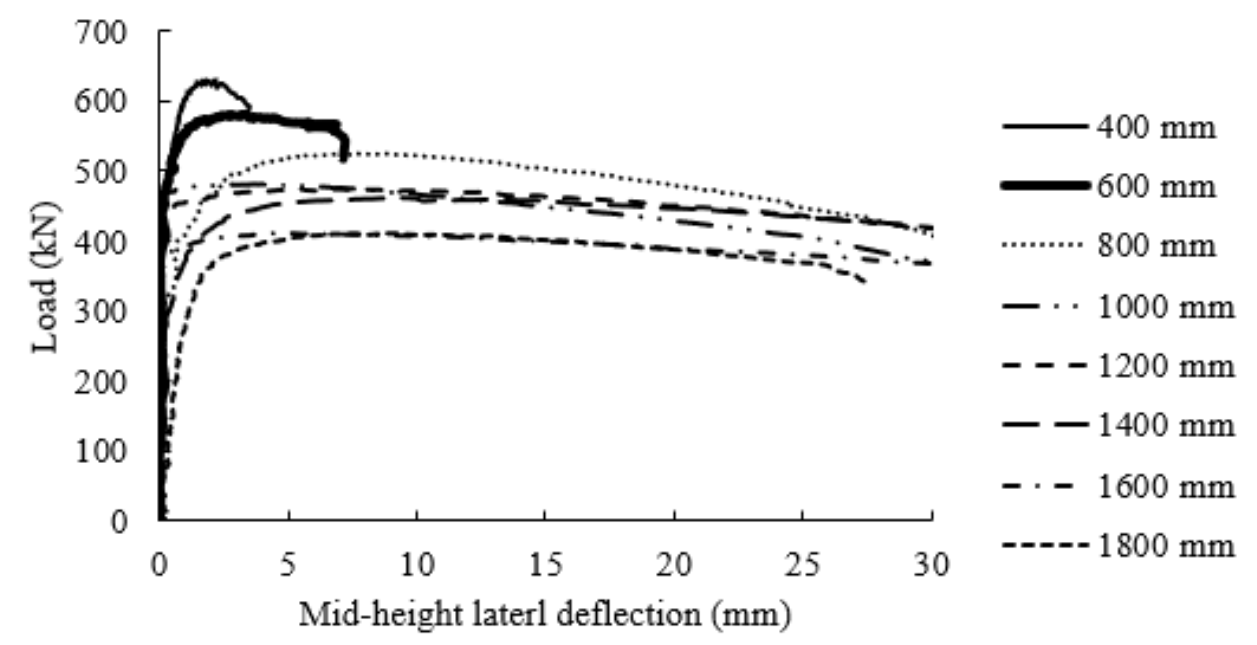

Fig. 7. Load vs lateral deflection

Ultimate capacity each group are summarized in Table. 1 and plotted in Fig. 8. Ultimate capacity was reduced significantly as slenderness ratio increased. Based on regression study, the ultimate capacity can be expressed by as Eq. 1 .

$$
\mathrm{P}=0.11 \lambda^{2}-213.47 \lambda+850.92
$$

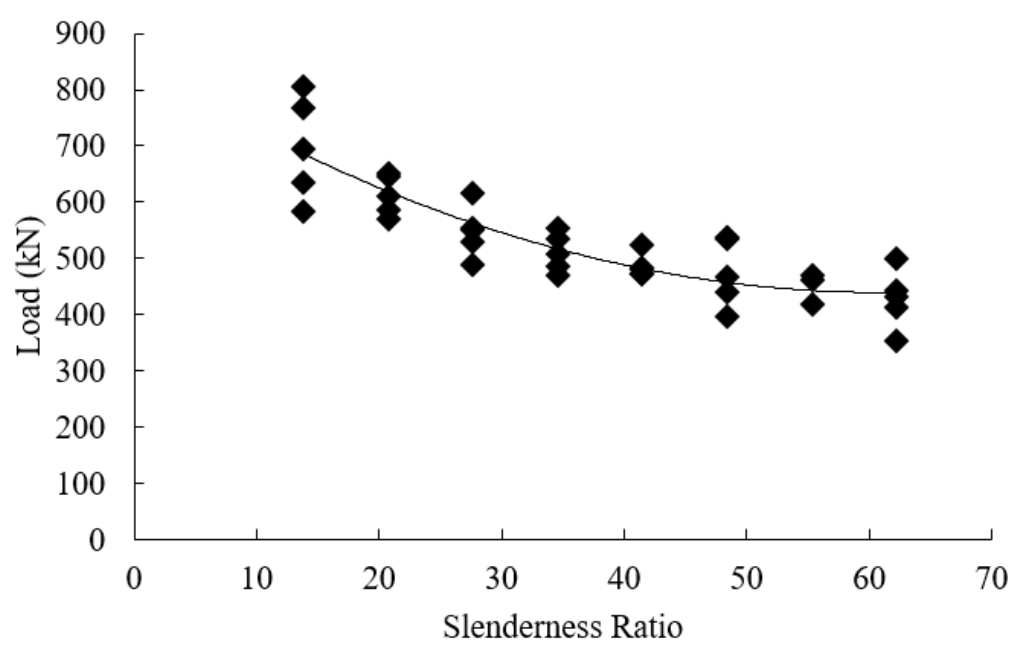

Fig. 8. Load capacity vs slenderness ratio

Where $\lambda$ is slenderness ratio,

$$
\lambda=\mathrm{L} / \sqrt{I / A}
$$

Where $\mathrm{L}$ is length of the column ( $\mathrm{mm})$; I and A are moment of inertia and area $\left(\mathrm{mm}^{4}\right)$ of column cross-section $\left(\mathrm{mm}^{2}\right)$. 
144 Fig. 9. shows load-longitudinal strain curves of each group of specimens. A, B, C and D represents the four surfaces of the tested columns. It can be seen that for group $400 \mathrm{~mm}$ and $600 \mathrm{~mm}$ columns, which failed by strength failure, all surfaces are under compression. For the rest groups, which failed in buckling, tensile strain was observed on the convex before ultimate

148 failure.

149

150

151

152

153

154

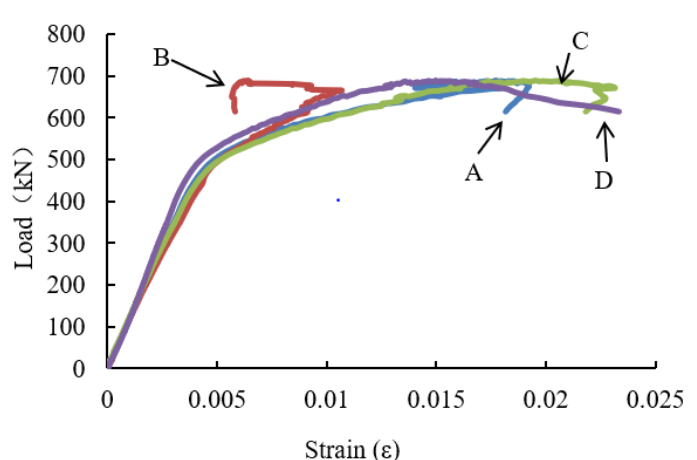

(a) 400-2

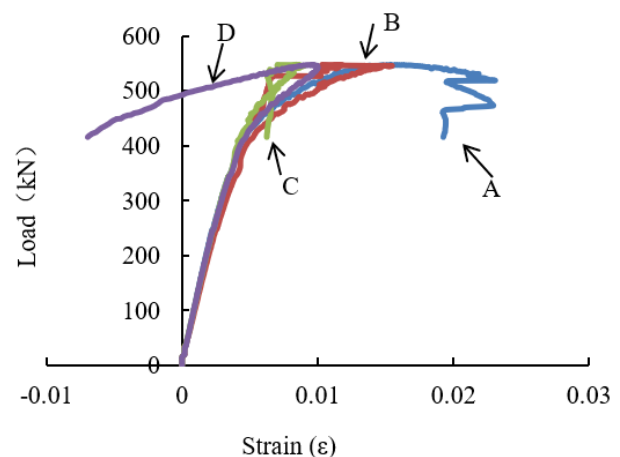

(c) $800-3$

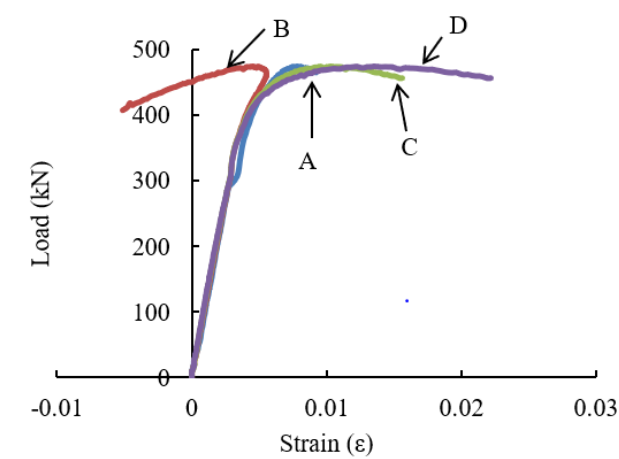

(e) 1200-6

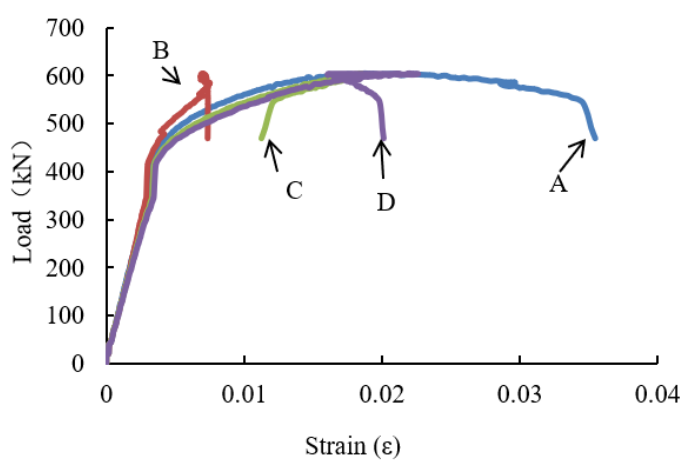

(b) 600-5

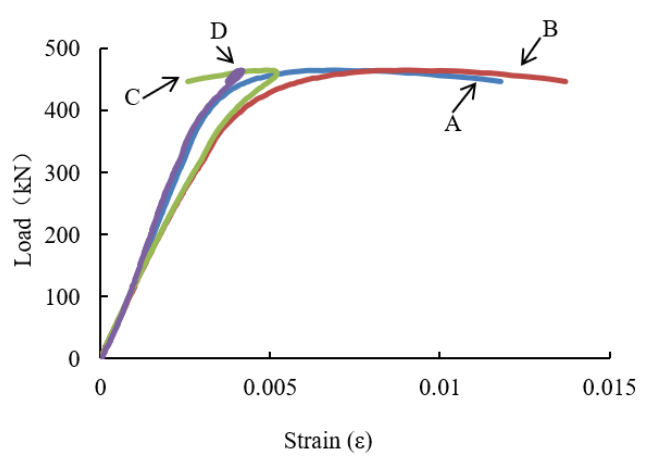

(d) 1000-2

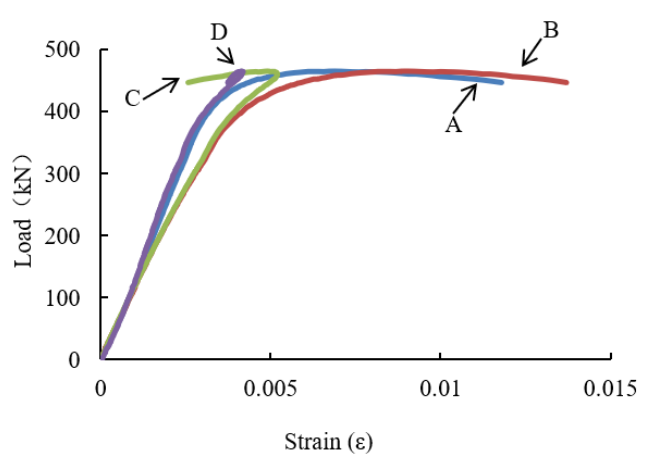

(f) 1400-2 


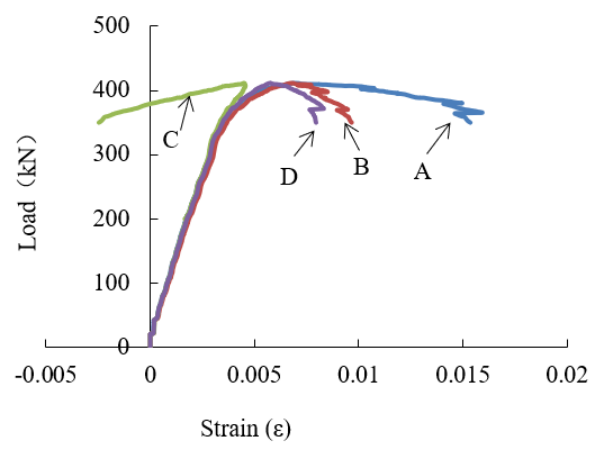

(g) 1600-3

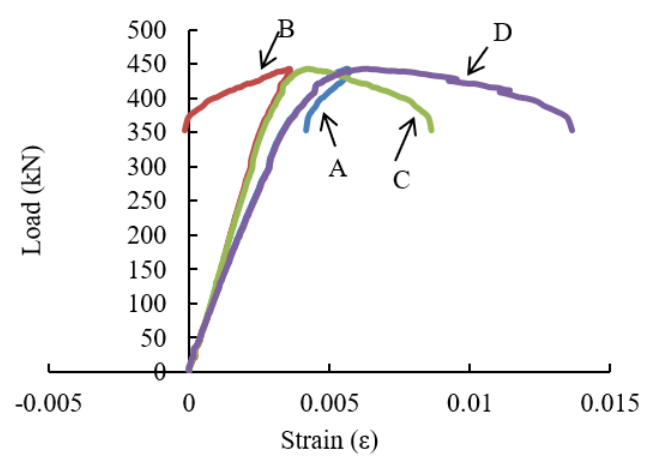

(h) 1800-1

Fig. 9. Load-strain response

Maximum longitudinal/transverse strain of each group are summarized in Table. 1. Maximum longitudinal and transverse strain of PBSL column were reduced as slenderness ratio increased. For short columns failure by strength failure, compressive strength of PBSL were fully developed as the measured longitudinal strain was close to the ultimate compressive strain. However, for columns failure in buckling, compressive property was not fully developed. Fig. 10 and 11 show the maximum strain versus slenderness ratio. The usable strain was significantly reduced as the length of column increased. Relationship between longitudinal/transverse strain and slenderness ratio can be summarized by statistical regression analysis, as shown in Eq. $3 \& 4$.

$$
\begin{aligned}
& \varepsilon_{l}=3.105 \lambda^{2}-467.6 \lambda+23533 \\
& \varepsilon_{t}=1.476 \lambda^{2}-283.2 \lambda+14350
\end{aligned}
$$

Where $\varepsilon_{l}$ is the maximum longitudinal strain at mid height; $\varepsilon_{t}$ is the maximum transverse strain at mid height; $\lambda$ is slenderness ratio. Longitudinal strain should not exceed ultimate compressive strain of PBSL material and transverse strain should not exceed product of ultimate compressive strain and Poisson's ratio.

Table 1: Test results

\begin{tabular}{ccccc}
\hline Length $(\mathrm{mm})$ & Slenderness ratio & Ultimate capacity $(\mathrm{kN})$ & Average $\varepsilon_{l \max }$ & Average $\varepsilon_{t \max }$ \\
\hline 400 & 13.8 & 685.06 & 0.0181 & 0.0102 \\
600 & 20.72 & 605.92 & 0.0153 & 0.0096 \\
800 & 27.64 & 538.34 & 0.0122 & 0.0082 \\
1000 & 34.58 & 502.26 & 0.0117 & 0.0056 \\
1200 & 41.50 & 478.48 & 0.0094 & 0.0049 \\
1400 & 48.45 & 467.02 & 0.0083 & 0.0035 \\
1600 & 55.35 & 440.73 & 0.0072 & 0.0032 \\
1800 & 62.26 & 423.20 & 0.0063 & 0.0024 \\
\hline
\end{tabular}




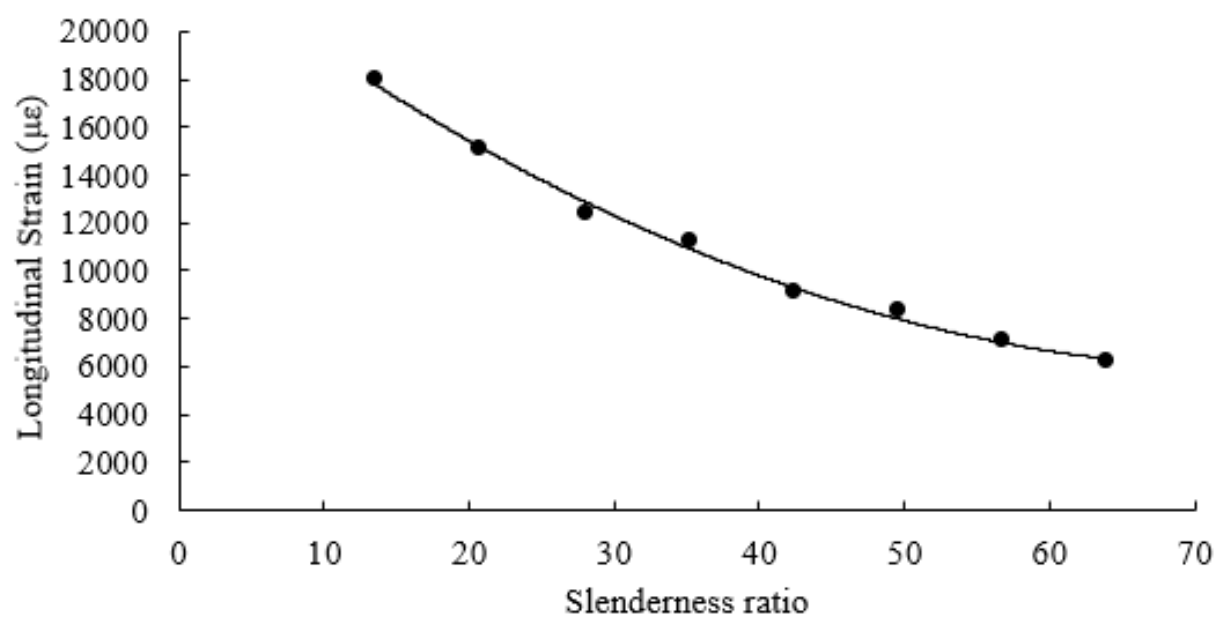

176
Fig. 10. Maximum longitudinal strain vs slenderness ratio

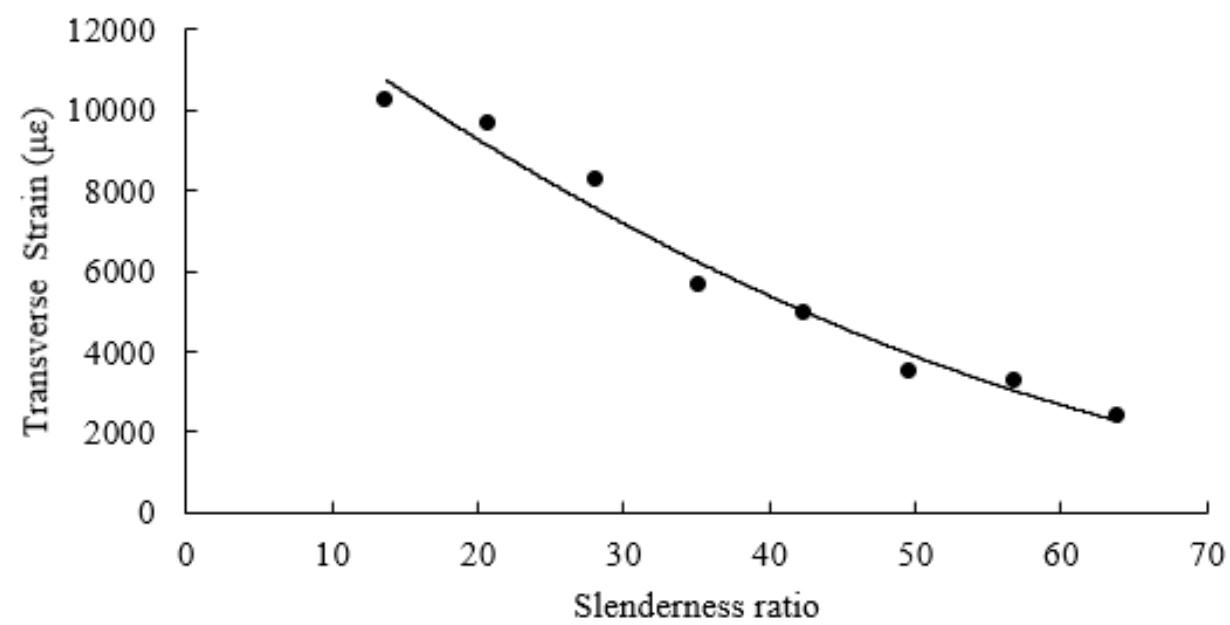

Fig. 11. Maximum transverse strain vs slenderness ratio

\section{Analytical Models}

\subsection{Euler's equation}

By using the method of neutral equilibrium, the critical buckling load can be solved from the governing differential equation for slight bent column configuration. In which, the column is assumed to be perfectly straight and material obeys Hooke's Law (elastic). Small lateral defection allows the curvature can be expressed as second derivative of the lateral deflection. The critical buckling load is solved through eigenvalue analysis as:

$$
P=\frac{n^{2} \pi^{2} E I}{L^{2}}
$$

Where $I$ is the moment of inertia of the cross-section and $L$ is length of the column.

The value of $P$ that corresponds to the smallest number $n(n=1)$ is the critical buckling load $\left(P_{c r}\right)$. Prediction of critical load with classic elastic Euler's equation and experiment results are plotted in Fig. 12. It shows the critical load is significantly overestimated for short and 
192 intermediate slender columns. However, as slenderness ratio approaching the elastic threshold $193\left(\lambda_{y}\right)$, reasonable agreement could be achieved.

$$
\lambda_{y}=\pi \sqrt{E / f_{y}}
$$

195 Where $E$ is the compressive elastic modulus, $f_{y}$ is the stress at proportional limit.

\subsection{Inelastic analysis}

\subsubsection{Tangent modulus and double modulus theory}

One of the assumptions used in Euler's equation is the material obeys Hooke's Law. However, this assumption is only valid for columns that are slender enough, so that buckling occurs before the proportional limit. For inelastically buckled columns, some fibers on the cross section yield before buckling occurs. As a result, additional load beyond proportional limit is resisted by a portion of the cross-section. The elastic modulus should be replaced by effective modulus, two widely used are tangent modulus and double modulus theory proposed by Engesser [28].

Other than assumptions addressed in elastic theory, it is assumed no strain reversal occurs during bending in tangent modulus theory. Tangent modulus $\left(E_{t}\right)$ of PBSL is determined as $3531.48 \mathrm{MPa}$ according to the stress-strain relation from the compressive property test. The critical load based on tangent modulus theory $\left(P_{t}\right)$ can be determined by Eq. 7 .

$$
P_{t}=\frac{\pi^{2} E_{t} I}{L^{2}}
$$

Double modulus theory, also referred as reduced modulus theory, was developed to address the strain reverse on the convex of the bended column. Fibers on convex tens to return to elastic stage, the section modulus is in between of elastic and tangent modulus. For rectangular sections, reduced modulus is

$$
E_{r}=\frac{4 E E_{t}}{\left(\sqrt{E}+{\sqrt{E_{t}}}^{2}\right.}
$$

The critical load based on double modulus theory $\left(P_{r}\right)$ can be determined by Eq. 9 .

$$
P_{r}=\frac{\pi^{2} E_{r} I}{L^{2}}
$$

Critical buckling load predicted using tangent/double modulus theory is plotted in Fig. 12. Both of the two approaches overestimate the capacity for short columns $(\lambda<20)$ and underestimate the capacity for relative long columns $(\lambda>40)$. 


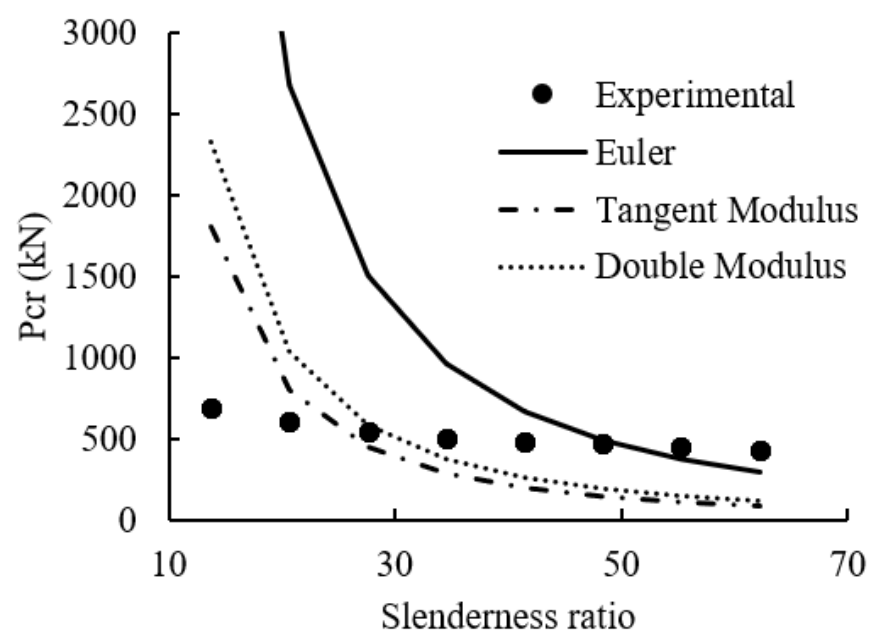

Fig. 12. Comparison of theoretical and experimental results

\subsubsection{Newlin-Gahagan approach}

Newlin-Gahagan approach [29] was developed for prediction of timber column buckling load. This approach has been proved to be efficient to predict inelastic buckling capacity of timber scrimber composite columns [30]. The critical stress $\left(f_{c r}\right)$ is expressed as a function of compressive stress $\left(f_{u}\right)$, proportional limit stress $\left(f_{y}\right)$, elastic threshold slenderness ratio $\left(\lambda_{y}\right)$ and actual slenderness ratio $(\lambda)$, all these properties could be achieved from the compressive stressstrain curve.

Critical buckling load can be calculated as the critical stress multiplied by the cross-section area. Prediction using Newlin-Gahagan approach is verified against experimental results, as shown in Fig. 13. It can be seen that the non-linear behavior can be accurately predicted by NewlinGahagan approach, however, for columns with large slenderness ratio, this method tends to underestimate the buckling load. The elastic threshold slenderness ratio can be used as a criterion to determine the applicability of this approach. For columns with slenderness ratio smaller than $\lambda_{y}$, whose ultimate capacity is dominated by inelastic behavior, the NewlinGahagan approach is applicable. Otherwise, the Euler's method is more suitable due to its reasonable accuracy and ease to apply. 


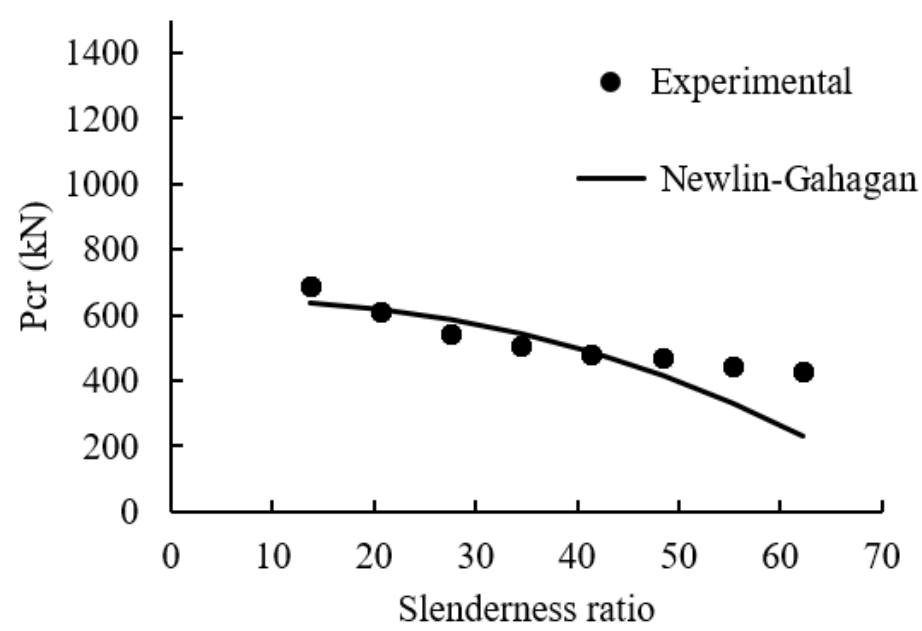

Fig. 13. Newlin-Gahagan approach

\section{Summary and Conclusion}

Axial compressive tests were conducted for PBSL column with various slenderness ratios. Failure mode, ultimate capacity and load-strain response were reported. For columns with slenderness ratio lower than 20 , strength failure was the typical failure model. No obvious later deflection was observed for the strength failed columns. For columns with slenderness ratio higher than 20, buckling dominates the failure mode. Ultimate capacity was reduced as the slenderness ratio increased. Significant lateral deflection was observed for buckling failed columns. According to the strain analysis, the compressive strength of PBSL could be almost fully developed for short columns $(\lambda<20)$. However, for longer columns failed by buckling, lower strain value was observed which indicated that the compressive strength was not fully developed.

Applicability of Classic Euler's method, tangent modulus theory, double modulus theory and Newlin-Gahagan approach were investigated regarding to ultimate capacity prediction of axial loaded PBSL columns. Analysis result shows inelasticity has significant effect on columns with a slenderness ratio lower than $\lambda_{y}$. Newlin-Gahagan approach provides good predictions of ultimate capacity of inelastically failed columns. For columns with a slenderness ratio higher than $\lambda_{y}$, the calssic Euler provides more accurate prediction than inelastic approaches.

Acknowledgement: This work was supported by the National Natural Science Foundation of China (51308301), the Natural Science Foundation of Jiangsu Province (No. BK20130978), the China Postdoctoral Science Foundation under Grant No. 2011M500930 and No. 2013T60544, the Foundation of the Doctoral Program of the Ministry of Education under Grant No. 20123204120012, and a Project Funded by the Priority Academic Program Development of Jiangsu Higher Education Institutions. The authors declare having no significant competing financial, professional, or personal interests that might have influenced the performance or presentation of the work described in this paper. The authors gratefully acknowledge the help from faculties, staff and students of Nanjing Forestry University.

\section{References}

[1] M. Ahmad, F. Kamke. Properties of parallel strand lumber from Calcutta bamboo (Dendrocalamus strictus). Wood Sci. Technol. 45(1) (2011) 63-72.

[2] P. Van der Lugt, Van den Dobbeksteen, J. Janssen. An environmental, economic and practical assessment of bamboo as a building material for supporting structures. Constr. 
Build. Mater. 20(9) (2006) 648-656.

[3] A. Porras, A. Maranon. Development and characterization of a laminate composite material from polylactic acid (PLA) and woven bamboo fabric. Composites Part B 43(7) (2012) 2782-2788.

[4] M. Mahdavi, P. Clouston, S. Arwade.(2010): Delvelopment of laminated bamboo lumber: review of processing, performance and economical considerations. J. Mater. Civ. Eng. 23(7) (2010) 1036-1042.

[5] H. Li, J. Su, A. Deeks, Q. Zhang, D. Wei, C. Yuan. Eccentric compression performance of parallel bamboo strand lumber columns. BioResour. 10(4) (2015) 7065-7080.

[6] B. Sharma, A. Gatóo, M. Bock, M. Ramage. Engineered bamboo for structural applications. Constr. Build. Mater. 81 (2015) 66-73.

[7] X. Li, M. Ashraf, H. Li, X. Zheng, A. Ameri. Behavior of parallel bamboo strand lumber under compression loading - an experimental study. MATEC Web of Conferences (2019) 275.

[8] P. Malanit, M. Barbu, A. Frühwald. Physical and mechanical properties of oriented strand lumber made from an Asian bamboo (Dendrocalamus asper Backer). Eur. J. Wood and Wood Prod. 69(1) (2010) 27-36.

[9] A. Kumar, T.Vlach, L. Laiblova, B. Hrouda, J. Tywoniak, P. Hajek. (2016): Engineered bamboo scrimber: Influence of density on the mechanical and water absorption properties. Constr. Build. Mater.127 (2016) 815-827.

[10] R. Kurt, E. Tomak. The effect of DMDHEU modification on physical and biological properties of parallel strand lumbers. Constr. Build. Mater. 195 (2019) 597-504.

[11] D.Huang, Y. Bian, A. Zhou, B.; Sheng. Experimental study on stress-strain relationships and failure mechanisms of parallel strand bamboo made from phyllostachys. Constr. Build. Mater.77 (2015) 130-138.

[12] H. Cui, M. Guan, Y. Zhu. The flexural characteristics of prestressed bamboo slivers reinforced parallel strand lumber (PSL). Key Engi. Mater. 517 (2012) 96-100.

[13] H.Zhang, H. Li, I. Corbi, O. Corbi, G. Wu, C. Zhao, T. Cao. AFRP influence on parallel bamboo strand lumber beams. Sensors 18(9) (2018) 2854.

[14] D. Huang, A. Zhou, Y. Bian, Y. Experimental and analytical study on the nonlinear bending of parallel strand bamboo beams. Constr. Buid. Mater. 44 (2013) 585-592.

[15] H. Li, Z. Qiu, G. Wu, O. Corbi, L. Wang, I. Corbi, C. Yuan. Slenderness ratio effect on eccentric compression performance of parallel strand bamboo lumber columns. J. Struct. Eng. ASCE., 145(8) (2019) 04019077

[16] D. Huang, Y. Bian, D. Huang, A. Zhou, B. Sheng. An ultimate-state-based-model for inelastic analysis of intermediate slenderness PSB columns under eccentrically compressive load. Constr. Build. Mater. 94 (2015) 306-314.

[17] X. Wang, A. Zhou, Y. Chui. Load-carrying capacity of intermediately slender parallel strand bamboo columns with a rectangular cross section under biaxial eccentric compression. Bioresour. 13(1) (2018) 313-330.

[18] G. Chen, H. Li, T. Zhou, C. Li, Y. Song, R. Xu. Experimental evaluation on mechanical performance of OSB webbedparallel strand bamboo I-joist with holes in the web. Constr. Build. Mater. 101 (2015) 91-98.

[19] J. Zhao, Z. Meng, Z. Jin, D. Chen, Y. Wu, W. Zhang. Bending properties of bamboo 

217.

[20] J. Zhou, D. Huang, Y. Song, C. Ni. Experimental investigation on embedding strength perpendicular to grain of parallel strand bamboo. Adv. in Mater. Sci. Eng. 8 (2018).

[21] P. Zhao, X. Zhang. Size effect of section on ultimate compressive strength parallel to grain of structural bamboo scrimber. Constr. Build. Mater. 200 (2019) 586-590.

[22] H. Li, Q. Zhang, D. Huang, A.J. Deeks. Compressive performance of laminated bamboo. Composites Part B 54(1) (2013) 319-328.

[23] H. Li, J. Su, Q. Zhang, A. Deeks, D. Hui. Mechanical performance of laminated bamboo column under axial compression. Composite Part B 79 (2015) 374-382.

[24] H. Li, G. Wu, Q. Zhang, J. Su. Mechanical evaluation for laminated bamboo lumber along two eccentric compression directions. J. wood sci. 62(6) (2016) 503-517.

[25] H. Li, G. Chen, Q. Zhang, M. Ashraf, B. Xu, Y. Li. Mechanical properties of laminated bamboo lumber column under radial eccentric compression. Constr. Build. Mater. 121 (2016) 644-652.

[26] H. Li, G. Wu, Q. Zhang, A.J. Deeks, J. Su. Ultimate bending capacity evaluation of laminated bamboo lumber beams. Constr. Build. Mater. 160 (2018) 365-375.

[27] ASTM. D198-15 Standard test methods of static tests of lumber in structural sizes. American Society for Testing and Materials. 2015.

[28] W. Chen, E. Lui.Structural Stablility: Theory and Implementation. Upper Saddle River, NJ: Prentice-Hall, Inc. (1987).

[29] J. Newlin, J. Gahagan. Tests of large timber columns and presentation of the forest products laboratory column formula. U. S Dep Agric Tech Bull. (1930).

[30] Z. Li, M. He, D. Tao, M. Li. Experimental buckling performance of scrimber composite columns under axial compression. Composite Part B 86 (2016) 203-213. 